\title{
EXACT LAGRANGIAN SUBMANIFOLDS, LAGRANGIAN SPECTRAL INVARIANTS AND AUBRY-MATHER THEORY
}

\author{
LINO AMORIM, YONG-GEUN OH, AND JOANA OLIVEIRA DOS SANTOS
}

ABSTRACT. We construct graph selectors for compact exact Lagrangians in the cotangent bundle of an orientable, closed manifold. The construction combines Lagrangian spectral invariants developed by $\mathrm{Oh}$ and results by Abouzaid about the Fukaya category of a cotangent bundle. We also introduce the notion of Lipschitz-exact Lagrangians and prove that these admit an appropriate generalization of graph selector. We then, following Bernard-Oliveira dos Santos, use these results to give a new characterization of the Aubry and Mañé sets of a Tonelli Hamiltonian and to generalize a result of Arnaud on Lagrangians invariant under the flow of such Hamiltonians.

\section{CONTENTS}

1. Introduction

2. Lipschitz-exact Lagrangian submanifolds

3. Action functional and Floer homology

3.1. Wrapped Floer homology

3.2. Floer homology of an exact Lagrangian and a fiber 11

4. Graph selectors

4.1. Choice of Hamiltonian $H$ adapted to $L$

4.2. Structure of the wave front of exact Lagrangians 13

4.3. Floer-theoretic graph selectors 14

5. Generalized graph selectors 16

6. Applications to Hamiltonian dynamics 17

7. Lipschitz continuity of the graph selector 19

References 23

\section{INTRODUCTION}

Consider the cotangent bundle $T^{*} M$ of a closed orientable $n$-manifold $M$. Let $\theta$ be the canonical Liouville one-form in $T^{*} M$. A Lagrangian embedding $\iota: N \rightarrow$ $T^{*} M$ is called exact if $\iota^{*} \theta$ is an exact one-form, that is, $\iota^{*} \theta=d S$ for some function $S: N \rightarrow \mathbb{R}$. We call any such function a Liouville primitive of the exact Lagrangian embedding $\iota: N \rightarrow T^{*} M$. We will denote

$$
L=\iota(N)
$$

Key words and phrases. Cotangent bundle, Lipschitz-exact Lagrangian submanifold, wrapped Fukaya category, (generalized) graph selector, Tonelli Hamiltonian, Aubry-Mather theory.

The first-named author was supported by EPSRC grant EP/J016950/1. The second-named author is supported by the IBS project \# IBS-R003-D1. 
as a submanifold of $T^{*} M$. In the present paper, we will distinguish the domain of an embedding $\iota$ and its image by different letters by exclusively denoting $L=\iota(N)$ while $N$ denotes an abstract $n$-manifold. We also denote by $i: L \hookrightarrow T^{*} M$ the inclusion map and by $h_{L}$ the function $h_{L}: L \rightarrow \mathbb{R}$ such that

$$
h_{L}(y)=S \circ \iota^{-1}(y), \quad y \in L .
$$

We will often omit the sub-index $L$ and denote $h=h_{L}$. This is nothing but the Liouville primitive for the inclusion $i: L \rightarrow T^{*} M$, that is $i^{*} \theta=d h_{L}$.

In many problems in the symplectic topology and Hamiltonian dynamics (see [PPS] and [BO2], for example) it is crucial to construct graph selectors for compact, exact Lagrangian submanifolds of $T^{*} M$.

Definition 1.1. Let $\iota: N \rightarrow T^{*} M$ be a compact, exact Lagrangian embedding with Liouville primitive $S$. A graph selector for $L$ is a Lipschitz function $f: M \rightarrow \mathbb{R}$ such that $f$ is differentiable on a dense open set $\mathcal{U} \subset M$ of full measure and for all points $q \in \mathcal{U}$ we have

$$
(q, d f(q)) \in L \text { and } f(q)=h_{L}(q, d f(q)) .
$$

The second equation in this definition is equivalent to

$$
\left.f \circ \pi\right|_{L}=h_{L}
$$

on $L$, where $\left.\pi\right|_{L}$ is the restriction to $L$ of the projection $\pi: T^{*} M \rightarrow M$.

There are several proofs that graph selectors exist for Lagrangians that are Hamiltonian isotopic to the zero-section. The proofs by Chaperon [C], PaternainPolterovich-Siburg [PPS] and Viterbo [V1] use generating functions in the sense of classical mechanics or micro-local analysis [Hor]. A more global definition of generating function of an exact Lagrangian submanifold is given in [Sik, V1], and it is proved by Laudenbach-Sikorav [LauS] that any Lagrangian Hamiltonian isotopic to the zero-section admits one. However a general compact exact Lagrangian submanifold is not known to have a generating function. Therefore this method of generating functions cannot be applied to general exact Lagrangian submanifolds (at least for now).

There is a second method to construct graph selectors developed by the secondnamed author in [Oh1] (and in other later literature), that uses Floer theory instead. This relies on being able to compute the Floer homology of $L$ with a cotangent fiber. Then one exploits the filtration present in the Floer complex to construct a spectral invariant which defines the function $f$, this is called the basic phase function. One of the main purposes of the present paper is to extend these constructions to general exact Lagrangian submanifolds and to explore their applications to Aubry-Mather theory.

One of the outstanding problems in symplectic geometry is the Arnold nearby Lagrangian conjecture which states that any compact exact Lagrangian is in fact Hamiltonian isotopic to the zero-section. Therefore a proof of this conjecture would immediately imply the existence of graph-selectors for any compact exact Lagrangian. This conjecture seems to be completely out of reach at the moment. However there have been spectacular advances in the understanding of this problem in the past few years, see [Ab2, FSS, N, Kra]. For example, it is now known that the projection from a compact exact Lagrangian to the zero-section is a homotopy equivalence. 
For our purposes the most important results are results by Abouzaid [Ab2] (using an argument due to [FSS]) and Abouzaid-Kragh [Kra] that prove the Floer homology between any compact, exact Lagrangian submanifold $L$ and a fiber $T_{q}^{*} M$ is isomorphic to $\mathbb{Z}$. This enables us to imitate constructions given in [Oh1, KO] and prove the following

Theorem 1.2. Let $L \subset T^{*} M$ be a closed smooth exact embedded Lagrangian submanifold. Then it admits a graph selector.

In the last couple of decades since the appearance of Eliashberg-Gromov's symplectic $C^{0}$-rigidity theorem [El], symplectic topology and Hamiltonian dynamics have witnessed many interesting $C^{0}$ phenomena. Some attempts to organize these phenomena in some conceptual way have been explored. For example, the second named author and Müller introduced the notion of $C^{0}$-Hamiltonian diffeomorphisms, called Hamiltonian homeomorphism (abbreviated as hameomorphism) in $[\mathrm{OM}]$, which has been further studied in later literature such as [Mu], [HLS].

In this paper we give a modest step in the direction of further understanding this $C^{0}$ nature of the symplectic world: we define Lipschitz-exact Lagrangians. This definition is partially motivated by the constructions in Aubry-Mather theory [BO2], where the study of such a class of Lagrangians is needed naturally. Imitating the construction of Hamiltonian homeomorphisms in $[\mathrm{OM}]$, we introduce them in Definition 2.4, by taking a completion of smooth exact Lagrangians in a suitably chosen topology. Roughly, a Lipschitz-exact Lagrangian consists of a smooth manifold $N$, a Lipschitz function $S$ on $N$ and a Lipschitz embedding

$$
\iota: N \longrightarrow T^{*} M,
$$

that can be approximated by a sequence of smooth exact Lagrangians $\iota_{k}: N \rightarrow$ $T^{*} M$.

The main result we prove about Lipschitz-exact Lagrangians is Theorem 5.2, which states that any compact Lipschitz-exact Lagrangian admits a generalized graph selector (Definition 5.1). This is a weakening of the notion of graph selector, which was introduced in [BO2] and plays a crucial role in our results on Hamiltonian dynamics.

We will give two applications of our results guaranteeing the existence of graph selectors, following the ideas of [BO1] and [BO2]. The first is in Aubry-Mather theory. Here we are interested in studying the dynamics of $C^{2}$ Tonelli Hamiltonians $H: T^{*} M \rightarrow \mathbb{R}$, which means convex with positive definite Hessian and superlinear in each fiber. These Hamiltonian are sometimes called optical.

We first introduce some notation we need to state the result. Denote by $\mathcal{G}$ the set

$$
\mathcal{G}:=\left\{\Gamma_{v} \mid v \in C^{1,1}(M)\right\},
$$

where $\Gamma_{v}:=\left\{(x, d v(x)) \in T^{*} M \mid x \in M\right\}$ and $C^{1,1}(M)$ are $C^{1}$ functions with Lipschitz differential. Let $\operatorname{Ham}\left(T^{*} M\right)$ be the set of Hamiltonian diffeomorphisms, Symp $p^{e}\left(T^{*} M\right)$ be the set of exact symplectic diffeomorphisms and define the sets

$$
\mathcal{H}:=\left\{\varphi\left(\Gamma_{v}\right) \mid \varphi \in \operatorname{Ham}\left(T^{*} M\right), \Gamma_{v} \in \mathcal{G}\right\}
$$

and

$$
\mathcal{E}:=\left\{\varphi\left(\Gamma_{v}\right) \mid \varphi \in \operatorname{Symp}^{e}\left(T^{*} M\right), \Gamma_{v} \in \mathcal{G}\right\}
$$

We define one more set,

$$
\mathcal{L}:=\left\{\text { compact Lipschitz-exact Lagrangians in } T^{*} M\right\},
$$


and remark that $\mathcal{H} \subset \mathcal{E} \subset \mathcal{L}$. The second inclusion is proved in Lemma 2.7. (We remark that in [BO2] the current $\mathcal{H}$ is denoted by $\mathcal{L}$. Here we reserve $\mathcal{L}$ for the set of Lipschitz-exact Lagrangians instead, which are defined in the present paper.)

Fix a $C^{2}$ Tonelli Hamiltonian $H: T^{*} M \rightarrow \mathbb{R}$ and an energy value $a \in \mathbb{R}$, for each manifold $L$ in $\mathcal{G}, \mathcal{H}, \mathcal{E}$ or $\mathcal{L}$ (or any other set of compact submanifolds of $T^{*} M$ ) we define the maximal invariant subset of $L \cap\{H=a\}$ by

$$
\mathcal{I}_{a}^{*}(L):=\bigcap_{t \in \mathbb{R}} \phi_{H}^{t}(L \cap\{H=a\}),
$$

where $\phi_{H}^{t}$ is the Hamiltonian flow of $H$. We define the critical value of $H$

$$
\alpha_{\mathcal{E}}(H):=\inf _{L \in \mathcal{E}} \max _{(q, p) \in L} H(q, p) .
$$

We now define the Aubry set $\mathcal{A}_{\mathcal{E}}^{*}(H)$ and the Mañé set $\mathcal{N}_{\mathcal{E}}^{*}(H)$ as

$$
\begin{aligned}
\mathcal{A}_{\mathcal{E}}^{*}(H) & :=\bigcap_{L \in \mathcal{E}, L \subset\left\{H \leq \alpha_{\mathcal{E}}(H)\right\}} \mathcal{I}_{\alpha_{\mathcal{E}}(H)}^{*}(L), \\
\mathcal{N}_{\mathcal{E}}^{*}(H) & :=\bigcup_{L \in \mathcal{E}, L \subset\left\{H \leq \alpha_{\mathcal{E}}(H)\right\}} \mathcal{I}_{\alpha_{\mathcal{E}}(H)}^{*}(L) .
\end{aligned}
$$

We can give analogous definitions, $\alpha_{\mathcal{S}}(H), \mathcal{A}_{\mathcal{S}}^{*}(H)$ and $\mathcal{N}_{\mathcal{S}}^{*}(H)$, by taking $L \in \mathcal{S}$, where $\mathcal{S}$ can be $\mathcal{G}, \mathcal{H}$ or $\mathcal{L}$. The classical definitions of these objects use $\mathcal{G}$. We would like to remark that it follows from [Be1] that $\alpha_{\mathcal{G}}(H)$ is in fact a minimum.

In [BO1] and [BO2] it is shown that if we use $\mathcal{H}$ we obtain the same objects and it is asked whether the same kind of result hold for the objects in $\mathcal{E}$.

Here, using the graph selector constructed in Theorem 1.2, we show that if we use $\mathcal{E}$ or even $\mathcal{L}$ we again obtain the same objects.

Theorem 1.3. Let $H: T^{*} M \rightarrow \mathbb{R}$ be a Tonelli Hamiltonian. Then

$$
\begin{aligned}
\alpha_{\mathcal{G}}(H) & =\alpha_{\mathcal{E}}(H)=\alpha_{\mathcal{L}}(H), \\
\mathcal{A}_{\mathcal{G}}^{*}(H) & =\mathcal{A}_{\mathcal{E}}^{*}(H)=\mathcal{A}_{\mathcal{L}}^{*}(H), \\
\mathcal{N}_{\mathcal{G}}^{*}(H) & =\mathcal{N}_{\mathcal{E}}^{*}(H)=\mathcal{N}_{\mathcal{L}}^{*}(H) .
\end{aligned}
$$

The reason for taking this approach is that definitions (1.3) and (1.4) are symplectically natural, meaning that the following result obtained by Bernard [Be] using variational methods becomes trivial:

Corollary 1.4. Let $H: T^{*} M \rightarrow \mathbb{R}$ be a Hamiltonian (not necessarily Tonelli). Then definitions (1.3) and (1.4) are equivariant under the action of $\operatorname{Symp}^{e}\left(T^{*} M\right)$. In other words given $\varphi \in \operatorname{Symp}^{e}\left(T^{*} M\right)$ we have

$$
\alpha_{\mathcal{E}}(H \circ \varphi)=\alpha_{\mathcal{E}}(H), \varphi\left(\mathcal{A}_{\mathcal{E}}^{*}(H \circ \varphi)\right)=\mathcal{A}_{\mathcal{E}}^{*}(H), \varphi\left(\mathcal{N}_{\mathcal{E}}^{*}(H \circ \varphi)\right)=\mathcal{N}_{\mathcal{E}}^{*}(H) .
$$

Proof. This easily follows from the definitions and the fact that if $L \in \mathcal{E}$ and $\varphi \in \operatorname{Symp}^{e}\left(T^{*} M\right)$, then $\varphi(L) \in \mathcal{E}$.

We observe that this result is only useful for the classical (using $\mathcal{G}$ ) Aubry and Mañé sets when both $H$ and $H \circ \varphi$ are Tonelli, that is, when Theorem 1.3 applies.

The next theorem is our second application.

Theorem 1.5. Suppose $M$ is connected and let $(N, \iota, S)$ be a compact Lipschitzexact Lagrangian in $T^{*} M$ in the sense of Definition 2.4. If $L=\iota(N)$ is invariant under the flow of a Tonelli Hamiltonian then $L \in \mathcal{G}$, that is, $L$ is a Lipschitz graph. 
A version of this theorem for smooth Lagrangians Hamiltonian isotopic to the zero section was proved by Arnaud [Arn]. It was then generalized in [BO2] to Lagrangians in $\mathcal{H}$.

\section{LIPSCHITZ-EXACT LAGRANGIAN SUBMANIFOLDS}

We start by recalling the notion of smooth exact Lagrangian submanifolds. We restrict ourselves to cotangent bundles, since that is the case we are interested in, but most of these notions make sense in general exact symplectic manifolds. Let $M$ be a closed smooth manifold of dimension $n$. We consider its cotangent bundle $X=T^{*} M$ with the usual symplectic form $\omega=-d \theta$, where $\theta$ is the canonical Liouville 1-form.

Definition 2.1. Let $N$ be a smooth $n$-manifold and $\iota: N \rightarrow T^{*} M$ be a smooth embedding. We say $(N, \iota)$ is an exact Lagrangian embedding if $\iota^{*} \theta$ is exact, and call its image $L=\iota(N)$ an exact Lagrangian submanifold of $T^{*} M$. We call the triple $(N, \iota, S)$ a Lagrangian brane where $S$ is a smooth function $S: N \rightarrow \mathbb{R}$ such that $d S=\iota^{*} \theta$. We call $S$ a Liouville primitive or just a primitive of $(N, \iota)$.

Definition 2.2. A diffeomorphism $\varphi: T^{*} M \rightarrow T^{*} M$ is called an exact symplectomorphism if $\varphi^{*} \theta-\theta$ is an exact 1 -form. We denote by $\operatorname{Symp} p^{e}\left(T^{*} M\right)$ the set of all exact symplectomorphisms.

In this paper we will be particularly interested in exact Lagrangians of the following form. Let $v: M \rightarrow \mathbb{R}$ be a smooth function and let

$$
\Gamma_{v}: M \longrightarrow T^{*} M, q \mapsto(q, d v(q))
$$

be the graph of its derivative. We have the following lemma

Lemma 2.3. Let $v$ be a smooth function and $\varphi \in \operatorname{Symp}^{e}\left(T^{*} M\right)$. Then the embedding

$$
\iota=\varphi \circ \Gamma_{v}: M \longrightarrow T^{*} M
$$

is an exact Lagrangian.

Proof. Since $\varphi$ is exact, there is $g: T^{*} M \rightarrow \mathbb{R}$ such that $\varphi^{*} \theta-\theta=d g$. We compute

$$
\iota^{*} \theta=\Gamma_{v}^{*}\left(\varphi^{*} \theta\right)=\Gamma_{v}^{*}(\theta+d g)=\Gamma_{v}^{*}(\theta)+d\left(\Gamma_{v}^{*}(g)\right) .
$$

By definition of $\theta$ we can easily see that $\Gamma_{v}^{*}(\theta)=d v$. Hence $\iota^{*} \theta=d\left(v+\Gamma_{v}^{*} g\right)$.

We now introduce a generalization of the notion of exact Lagrangian that we call Lipschitz-exact Lagrangians.

Definition 2.4. Let $N$ be a smooth $n$-manifold, $\iota: N \rightarrow T^{*} M$ be an injective continuous map and let $S: N \rightarrow \mathbb{R}$ be a continuous function. We say the triple $(N, \iota, S)$ is a Lipschitz-exact Lagrangian brane if there are equi-Lipschitz sequences $S_{k}: N \rightarrow \mathbb{R}$ of smooth functions and $\iota_{k}: N \rightarrow T^{*} M$ of smooth embeddings such that

(a) $\iota_{k}^{*} \theta=d S_{k}$,

(b) $S_{k} \rightarrow S$ and $\iota_{k} \rightarrow \iota$ uniformly (in the $C^{0}$ topology).

We call any such sequence $\left(N, \iota_{k}, S_{k}\right)$ an approximating sequence of the Lipschitzexact Lagrangian brane $(N, \iota, S)$. 
Note that as consequence of the definition, if $(N, \iota, S)$ is a Lipschitz-exact Lagrangian brane, then $\iota$ and $S$ are Lipschitz. With this definition we can also see that $S$ is a primitive of the pull-back of $\theta$ in the sense of the next proposition.

Proposition 2.5. Let $(N, \iota, S)$ be a Lipschitz-exact Lagrangian brane. Then

$$
\iota^{*} \theta=d S
$$

as a one-form in $L^{\infty}$. In particular $\iota^{*} \omega=0$ as a two-form in $L^{\infty}$.

Proof. We assume $N$ is orientable. (Otherwise we just use the odd differential forms in the sense of de Rham [dR] for the arguments below and so we will focus on the case of orientable $N$.)

We fix a Riemannian metric on $N$ and denote by vol the associated volume form. We first note that both sides of the equation define well-defined currents since $\iota$ and $S$ are Lipschitz functions and so are in $W^{1, \infty}$ (see [EG, Section 4.2.3, Theorem 4]). By a standard theorem (see [EG, Section 6.2, Theorem 1], for example), $d S$ equals its weak derivative almost everywhere. Therefore it is enough to prove that the weak derivative (or equivalently the derivative as a current) is the same as $\iota^{*} \theta$.

Let $\eta$ be any smooth $(n-1)$-form. Then the weak derivative $d S$ satisfies

$$
\begin{aligned}
\int_{N} d S \wedge \eta & =-\int_{N} S d \eta=-\lim _{k \rightarrow \infty} \int_{N} S_{k} d \eta \\
& =\lim _{k \rightarrow \infty} \int_{N} d S_{k} \wedge \eta=\lim _{k \rightarrow \infty} \int_{N} 1_{k}^{*} \theta \wedge \eta .
\end{aligned}
$$

Here we use the uniform convergence of $S_{k}$ to $S$ for the second equality. Without loss of generality, we assume that $\operatorname{supp} \eta$ is contained in a Darboux neighbohood $U$ equipped with canonical coordinates $(q, p), q=\left(q_{1}, \ldots, q_{n}\right), p=\left(p_{1}, \ldots, p_{n}\right)$ for which $\theta=p d q=\sum_{i=1}^{n} p_{i} d q_{i}$. We now express $\iota_{k}^{*} \theta=p \circ \iota_{k} \cdot d\left(q \circ \iota_{k}\right)$ and rewrite

$$
\int_{N} 1_{k}^{*} \theta \wedge \eta=\int_{U} p \circ \iota_{k} \cdot d\left(q \circ \iota_{k}\right) \wedge \eta
$$

We will show

$$
\int_{U} p \circ \iota_{k} \cdot d\left(q \circ \iota_{k}\right) \wedge \eta \rightarrow \int_{U} p \circ \iota \cdot d(q \circ \iota) \wedge \eta
$$

This is where the hypothesis that $\iota_{k}$ is equi-Lipschitz enters in a crucial way: it first implies that $q \circ \iota_{k}, p \circ \iota_{k}$ are also equi-Lipschitz. We rewrite

$$
\begin{aligned}
& \int_{U} p \circ \iota_{k} \cdot d\left(q \circ \iota_{k}\right) \wedge \eta-\int_{U} p \circ \iota \cdot d(q \circ \iota) \wedge \eta \\
= & \int_{U}\left(p \circ \iota_{k}-p \circ \iota\right) \cdot d\left(q \circ \iota_{k}\right) \wedge \eta+\int_{U} p \circ \iota \cdot d\left(q \circ \iota_{k}-q \circ \iota\right) \wedge \eta .
\end{aligned}
$$

For the first integral, we have the bound

$$
\left|\int_{U}\left(p \circ \iota_{k}-p \circ \iota\right) \cdot d\left(q \circ \iota_{k}\right) \wedge \eta\right| \leq\left\|p \circ \iota_{k}-p \circ \iota\right\|_{L^{\infty}} \int_{U}\left|d\left(q \circ \iota_{k}\right)\right| \cdot|\eta| \operatorname{vol} .
$$

Since $q \circ \iota_{k}$ are equi-Lipschitz, $d\left(q \circ \iota_{k}\right)$ have an uniform bound on $L^{\infty}$-norm. Therefore there exists $C>0$ independent of $k$ such that

$$
\int_{U}\left|d\left(q \circ \iota_{k}\right)\right| \cdot|\eta| \mathrm{vol} \leq C
$$


for all $k$. Then by the uniform convergence of $p \circ \iota_{k} \rightarrow p \circ \iota$, we have derived

$$
\int_{U}\left(p \circ \iota_{k}-p \circ \iota\right) \cdot d\left(q \circ \iota_{k}\right) \wedge \eta \rightarrow 0
$$

Next, we consider the integral

$$
\int_{U} p \circ \iota \cdot d\left(q \circ \iota_{k}-q \circ \iota\right) \wedge \eta
$$

Again using the uniform bound on the $W^{1, \infty}$-norm of $q \circ \iota_{k}$, we can choose a subsequence, still denoted by $q \circ \iota_{k}$, weakly-^ converging to $q \circ \iota$ in $W^{1, \infty}$. Therefore

$$
\int_{U} p \circ \iota \cdot d\left(q \circ \iota_{k}-q \circ \iota\right) \wedge \eta=(-1)^{n-1} \int_{U}\left(\eta \wedge(p \circ \iota) d\left(q \circ \iota_{k}\right)-\eta \wedge(p \circ \iota) d(q \circ \iota)\right) \rightarrow 0 .
$$

This proves the convergence

$$
\int_{U} p \circ \iota_{k} \cdot d\left(q \circ \iota_{k}\right) \wedge \eta \rightarrow \int_{U} p \circ \iota \cdot d(q \circ \iota) \wedge \eta=\int_{N} \iota^{*} \theta \wedge \eta
$$

after taking a subsequence.

Combining 2.2 and 2.3, we have proved

$$
\int_{N} d S \wedge \eta=\int_{N} \iota^{*} \theta \wedge \eta
$$

for all smooth $(n-1)$-form $\eta$, that is, the weak derivative of $S$ is given by $d S=\iota^{*} \theta$.

In particular $d\left(\iota^{*} \theta\right)=0$ as a current. On the other hand by the standard identity $d \varphi^{*}=\varphi^{*} d$ acting on the set of currents for the Lipschitz map $\varphi$ (see e.g., [Fe, Section 4.4.1]), we obtain $\iota^{*} d \theta=d\left(\iota^{*} \theta\right)$ and hence $\iota^{*} \omega=-\iota^{*}(d \theta)=0$ as a current. This finishes the proof.

The following proposition is proved in the same way as Proposition 2.5. It is also proved in Proposition 2 in [BO2] in a slightly different setting.

Proposition 2.6. Let $(N, \iota, S)$ be an Lipschitz-exact Lagrangian brane and let $c:[0,1] \rightarrow N$ be a Lipschitz curve, then

$$
\int_{\llcorner\circ c} \theta=S(c(1))-S(c(0))
$$

Proof. We follow the proof above, with $S \circ c$ and $(\iota \circ c)^{*} \theta$ instead of $S$ and $\iota^{*} \theta$. All the arguments apply since $S \circ c$ and $\iota \circ c$ are still Lipschitz.

Our main example of Lipschitz-exact Lagrangian branes are the elements of $\mathcal{E}$.

Lemma 2.7. Let $v: M \rightarrow \mathbb{R}$ be a $C^{1,1}$ function and let $\varphi: T^{*} M \rightarrow T^{*} M$ be an exact symplectomorphism with $\varphi^{*} \theta-\theta=d g$. Then $\iota: M \rightarrow T^{*} M$ given by $\iota=\varphi \circ \Gamma_{v}$ is Lipschitz with Lipschitz primitive $S=v+\Gamma_{v}^{*} g$. That is, the triple $(M, \iota, S)$ is a Lipschitz-exact Lagrangian brane. In other words, $\mathcal{E} \subset \mathcal{L}$.

Proof. Let $v_{k}$ be a sequence of smooth functions converging to $v$ in $C^{1}(M)$ and bounded in $W^{2, \infty}(M)$. Take $\iota_{k}=\varphi \circ \Gamma_{v_{k}}$ and $S_{k}=v_{k}+\Gamma_{v_{k}}^{*} g$. Lemma 2.3 now implies that $\iota_{k}^{*} \theta_{k}=d S_{k}$. Moreover the $S_{k}$ are equi-Lipschitz and converge uniformly to $S$ and similarly the sequence $\iota_{k}$ is equi-Lipschitz and converges uniformly to $\iota$. 
We now discuss possible alternative definitions of Lipschitz-exact Lagrangians. Instead of requiring that the sequences $\iota_{k}$ and $S_{k}$ be equi-Lipschitz, we could have required that both $\iota_{k}$ and $S_{k}$ converge to $\iota$ and $S$ in $C^{1,1}$ respectively. But this definition would be too strong and we wouldn't be able to prove Lemma 2.7 (since smooth functions are not dense in $\left.C^{1,1}(M)\right)$.

On the other direction we could have dropped the equi-Lipschitz condition. Then one would get a definition of Lagrangian analogous to those from $C^{0}$-symplectic geometry. We will not do this here since for our purposes, namely the existence of graph selectors, this seems too weak. Also, for the applications to Aubry-Mather theory our definition suffices.

However, it would be interesting to study this more general definition. One could ask if, whenever $\iota$ is a smooth embedding, then $\iota: N \rightarrow T^{*} M$ is an exact Lagrangian in the usual sense. This would be a version of Eliashberg-Gromov $C^{0}$ rigidity for exact Lagrangians. We observe that Proposition 2.5 implies this is the case for our more restrictive definition. For this more general definition another interesting question would be the following.

Question 2.8. Let $\iota_{k}$ and $S_{k}$ be uniformly convergent sequences. Assume a normalization condition on the $S_{k}$, say $S_{k}\left(x_{0}\right)=0$ for all $k$ at a given point $x_{0} \in N$. Will the uniform limit of $\iota_{k}$ determine the limit of $S_{k}$ ?

This question would be a counterpart of the fundamental uniqueness result in topological Hamiltonian dynamics given in [OM, V2, Oh4, BS]. We observe that for our definition of Lipschitz-exact Lagrangian, Proposition 2.5 answers this question affirmatively. We hope to come back to the study of these questions elsewhere.

\section{Action functional and Floer homology}

3.1. Wrapped Floer homology. In this subsection we briefly review some aspects of wrapped Floer cohomology as in [Ab2]. Our conventions for the action functional and gradings will differ from the ones in [Ab2], we will instead follow [Oh1], and naturally obtain a homology complex. In this section and the next two, we will exclusively consider a Lagrangian submanifold $L$ as a subset $T^{*} M$ because all constructions will depend only on the image.

For a given $(N, \iota, S)$, we denote

$$
L=\iota(N), \quad h=S \circ \iota^{-1} .
$$

Then by definition, we have $i^{*} \theta=d h$ on $L$.

Recall that $T^{*} M$ is a Liouville manifold, that means there is a (codimension zero) submanifold with boundary $K \subseteq T^{*} M$, such that $\theta$ restricts to a contact form on $\partial K$ and there is a diffeomorphism

$$
T^{*} M \backslash K \simeq[1,+\infty) \times \partial K
$$

identifying $\theta$ with $\left.r \cdot \theta\right|_{\partial K}$, where $r$ is the coordinate in $[1,+\infty)$.

To see this we can fix a metric on $M$ and take $K=D_{1}^{*} M$, where

$$
D_{\rho}^{*} M=\left\{(q, p) \in T^{*} M \mid\langle p, p\rangle \leq \rho^{2}\right\} .
$$

We will only consider Lagrangian submanifolds which are compact or asymptotically conic: 
Definition 3.1. An (embedded) Lagrangian submanifold $L$ in $T^{*} M$ is called asymptotically conic if, for $K$ as in (3.1), if we denote $L^{K}:=L \cap K$, then $\partial L^{K}$ is Legendrian in $\partial K$ and we have a diffeomorpphism $L \backslash L^{K} \simeq[1,+\infty) \times L^{K}$.

In fact, the only non compact Lagrangians we shall consider in the present paper are cotangent fibers $T_{q}^{*} M, q \in M$, which are obviously conic.

We need to choose some auxiliary data to define the wrapped Floer cohomology. We fix a Hamiltonian function $H: T^{*} M \rightarrow \mathbb{R}$ quadratic at infinity, which means it is equal to $r^{2}$ on $T^{*} M \backslash K$. Note we only impose a restriction on $H$ outside of $K$ and so these Hamiltonians are different from the Tonelli Hamiltonians we consider in Section 7.

Given a pair of Lagrangian submanifolds $\left(L_{0}, h_{0}\right),\left(L_{1}, h_{1}\right)$ we define a function on $\mathcal{P}\left(L_{0}, L_{1}\right)$ the space of paths $\gamma:[0,1] \rightarrow T^{*} M$ satisfying $\gamma(0) \in L_{0}, \gamma(1) \in L_{1}$ :

$$
\mathcal{A}_{H}(\gamma)=\int_{[0,1]}\left(\gamma^{*} \theta-H \circ \gamma d t\right)+h_{0}(\gamma(0))-h_{1}(\gamma(1)) .
$$

We call this function the action functional.

Remark 3.2. Here we use the sign convention of [Oh1, KO] for the action functional, so that when $h_{1}=h_{0}=0$, the action functional becomes the classical action functional in the mechanics literature. We remark that this definition is the negative of the one used in [Ab2] but coincides with that of [Be]. We regard the definition of the natural homological complex in Floer theory as the homological complex and then by reversing the flow, we identify the corresponding complex with the cohomological one. In this way, our definition of wrapped Floer homology can be identified with that of the cohomology in [Ab2].

The first variation formula of this action functional is given by

$$
d \mathcal{A}_{H}(\gamma)(\xi)=\int_{[0,1]} \omega\left(\dot{\gamma}-X_{H}(t, \gamma(t)), \xi(t)\right) d t,
$$

where $X_{H}$ is the Hamiltonian vector field associated to $H$, that is $\omega\left(X_{H}, \cdot\right)=d H$.

The following proposition immediately follows from (3.3).

Proposition 3.3. Let $\gamma \in \mathcal{P}\left(L_{0}, L_{1}\right)$, then $\gamma$ is a critical point of $\mathcal{A}_{H}$ if and only if it satisfies $\dot{\gamma}(t)=X_{H}(\gamma(t))$. We denote by $\mathcal{X}\left(L_{0}, L_{1}\right)$ the set of critical points $p f$ $\mathcal{A}_{H}$.

Given the conditions imposed on the Lagrangians we have the following lemma (see $[\mathrm{Ab} 1]$ ).

Lemma 3.4. Given $a \in \mathbb{R}$ denote by $\mathcal{X}_{\leq a}\left(L_{0}, L_{1}\right)$ the set of critical points $\gamma$ of $\mathcal{A}_{H}$ with $\mathcal{A}_{H}(\gamma) \leq a$. This set is compact and after generic perturbation of $L_{0}$ (or $L_{1}$ ) it is finite.

In order to define wrapped Floer homology as a $\mathbb{Z}$-graded abelian group we need to impose some topological restrictions on the Lagrangians.

From now on we assume that $M$ is orientable. Then we choose a volume form in $M$ and we complexify it (using a compatible almost complex structure). This gives a quadratic complex volume form $\eta$ on $T^{*} M$ (see details in [Ab2] and Section 11 of [Se]). For each point $p$ in a Lagrangian $L$, we can evaluate $\eta /|\eta|$ on a basis for $T_{p} L$. This is independent of the choice of basis and therefore defines a map

$$
\frac{\eta}{|\eta|}: L \longrightarrow S^{1} \text {. }
$$


If the Maslov class of $L$ vanishes (see Section 11 of [Se] for details) we can choose a lift of this map to a real-valued function. We call such choice a grading on $L$. By choosing gradings on $L_{0}$ and $L_{1}$ we can assign a Maslov index to each element $\gamma \in \mathcal{X}\left(L_{0}, L_{1}\right)$ which we denote by $|\gamma| \in \mathbb{Z}$.

We define the wrapped Floer complex as

$$
C W_{*}\left(L_{0}, L_{1}\right)=\bigoplus_{i} C W_{i}\left(L_{0}, L_{1}\right)=\bigoplus_{i} \bigoplus_{\begin{array}{c}
x \in \mathcal{X}\left(L_{0}, L_{1}\right) \\
|x|=i
\end{array}} x \cdot \mathbb{Z} .
$$

That is $C W_{i}\left(L_{0}, L_{1}\right)$ is the free abelian group generated by Hamiltonian chords of Maslov index $i$.

Next we define the Floer differential. For this we need to consider a family $\left\{J_{t}\right\}_{t \in[0,1]}$ of almost complex structures on $T^{*} M$ compatible with $\omega$. Additionally we assume that in the complement of $K$ each complex structure $J$ satisfies

$$
\theta \circ J=d r .
$$

We consider the Cauchy-Riemann equation in the space of maps $u: \mathbb{R} \times[0,1] \rightarrow$ $T^{*} M$

$$
\left\{\begin{array}{l}
\frac{\partial u}{\partial \tau}+J_{t}\left(\frac{\partial u}{\partial t}-X_{H}(u)\right)=0, \\
u(\tau, 0) \in L_{0}, u(\tau, 1) \in L_{1} .
\end{array}\right.
$$

Given $x_{0}, x_{1} \in \mathcal{X}\left(L_{0}, L_{1}\right)$ we denote by $\mathcal{M}\left(x_{0}, x_{1}\right)$ the set of maps $u$ satisfying the above equation and converging (exponentially) to $x_{0}$ at $-\infty$ and to $x_{1}$ at $+\infty$, quotiented by translations on the $\tau$-direction. For generic $\left\{J_{t}\right\}$, the moduli spaces $\mathcal{M}\left(x_{0}, x_{1}\right)$ are smooth manifolds of dimension $\left|x_{0}\right|-\left|x_{1}\right|-1$.

To define orientations on these moduli spaces coherently we need to impose one more topological restriction on the Lagrangians. Denote by $b=\pi^{*} w_{2}(T M) \in$ $H^{2}\left(T^{*} M, \mathbb{Z}_{2}\right)$ the pullback of the second Stiefel-Whitney class of $M$. We say a Lagrangian $L$ is relatively spin if $\left.b\right|_{L}=w_{2}(T L)$ and a choice of relative spin structure is defined to be a spin structure on the vector bundle $\left.T L \oplus \pi^{*}(T M)\right|_{L}$.

Relative spin structures on $L_{0}$ and $L_{1}$ then determine orientations on the moduli spaces $\mathcal{M}\left(x_{0}, x_{1}\right)$ (see $[\mathrm{Ab} 1]$ for details).

When $\left|x_{0}\right|=\left|x_{1}\right|+1, \mathcal{M}\left(x_{0}, x_{1}\right)$ is a zero-dimensional oriented manifold and Gromov compactness implies that it is compact. Hence we define $\# \mathcal{M}\left(x_{0}, x_{1}\right)$ as the signed count of elements in $\mathcal{M}\left(x_{0}, x_{1}\right)$. Finally we define

$$
\partial: C W_{i}\left(L_{0}, L_{1}\right) \rightarrow C W_{i-1}\left(L_{0}, L_{1}\right)
$$

as

$$
\partial\left(x_{0}\right)=\sum_{\substack{x_{1} \in \mathcal{X}\left(L_{0}, L_{1}\right) \\\left|x_{0}\right|=\left|x_{1}\right|+1}} \# \mathcal{M}\left(x_{0}, x_{1}\right) \cdot x_{1} .
$$

A standard argument implies the following

Lemma 3.5. The map $\partial$ is a differential, i.e. $\partial^{2}=0$.

This differential has one additional property which follows from the following standard lemma. (See [Oh1] or [Ab2] for the proof.)

Lemma 3.6. If $\mathcal{M}\left(x_{0}, x_{1}\right)$ is non-empty, then

$$
\mathcal{A}_{H}\left(x_{0}\right) \geq \mathcal{A}_{H}\left(x_{1}\right) \text {. }
$$


We define a filtration $\nu: C W_{*}\left(L_{0}, L_{1}\right) \rightarrow \mathbb{R}$ as follows: given $\alpha=\sum_{x} a_{x} x \in$ $C W_{*}\left(L_{0}, L_{1}\right)$ we take $\nu(\alpha)=\max _{a_{x} \neq 0}\left\{\mathcal{A}_{H}(x)\right\}$. The above lemma implies that

$$
C W_{*}^{\leq a}\left(L_{0}, L_{1}\right)=\left\{\alpha \in C W\left(L_{0}, L_{1}\right) \mid \nu(\alpha) \leq a\right\}
$$

is a subcomplex of $C W_{*}\left(L_{0}, L_{1}\right)$.

We define the wrapped Floer homologies

$$
H W_{*}\left(L_{0}, L_{1}\right)=\operatorname{ker} \partial / \operatorname{Im} \partial
$$

and

$$
H W_{*}^{\leq a}\left(L_{0}, L_{1}\right)=\operatorname{ker} \partial \leq a / \operatorname{Im} \partial \leq a
$$

where $\partial \leq a$ is the restriction of $\partial$ to the subcomplex $C W_{*}^{\leq a}\left(L_{0}, L_{1}\right)$. Note that the inclusion $j_{a}: C W_{*}^{\leq a}\left(L_{0}, L_{1}\right) \hookrightarrow C W_{*}\left(L_{0}, L_{1}\right)$ induces a map

$$
\left(j_{a}\right)_{*}: H W_{*}^{\leq a}\left(L_{0}, L_{1}\right) \rightarrow H W_{*}\left(L_{0}, L_{1}\right) \text {. }
$$

One can be also define the wrapped Floer cohomology $H W^{*}\left(L_{1}, L_{0}\right)$ and its filtration $H W_{\geq a}^{*}\left(L_{1}, L_{0}\right)$ following the canonical construction of cohomology. This can be canonically identified with the complex obtained by 'reversing the flow'. In the present case, the latter construction then will lead to the wrapped Floer cohomology defined in [Ab2].

3.2. Floer homology of an exact Lagrangian and a fiber. The wrapped Floer complexes defined in the previous subsection for each pair of Lagrangians can be combined to define an $\mathrm{A}_{\infty}$-category, $\mathcal{W}\left(T^{*} M\right)$ known as the wrapped Fukaya category of $T^{*} M$. The full details of this construction are intricate and can be found in $[\mathrm{AS}]$ and $[\mathrm{Ab} 1]$.

Remark 3.7. We would like to note that in [Ab1] the complex $C W^{*}\left(L, L^{\prime}\right)$ is associated to the space of paths running from $L$ to $L^{\prime}$ which is opposite to our convention. Our enumeration of the $L_{i}$ 's in the pair $C W^{*}\left(L_{i}, L_{i-1}\right)$ is the opposite to the one given in [Ab1]. Our present convention is consistent with that of [FOOO]. (More specifically see Section 2.3 [FOOO] and also Remark 3.2 of the present paper.)

The work of Abouzaid [Ab1], [Ab2] gives a complete description of the category $\mathcal{W}\left(T^{*} M\right)$. In $[\mathrm{Ab} 1]$ the author proves that any cotangent fiber $T_{q}^{*} M$ generates this category. We will not make use of this statement in its entirety, we will just use a particular consequence of this fact proved in Appendix $\mathrm{C}$ of [Ab2].

Theorem 3.8 (Abouzaid). Assume $M$ is orientable and let $L$ be an exact, compact Lagrangian with vanishing Maslov class, then

(a) $L$ is relatively spin;

(b) the Floer cohomology $H W^{*}\left(T_{q}^{*} M, L\right) \simeq \mathbb{Z}$ is free of rank 1 .

Note that a cotangent fiber $T_{q}^{*} M$ has vanishing Maslov class and it is relatively spin since it is contractible and $b_{\mid T_{q}^{*} M}=0$. Therefore part (a) implies that the Floer cohomology in (b) is well-defined. In fact the proof of the above theorem requires an extension of the wrapped Fukaya category that includes local systems on the Lagrangians - this is carried out in [Ab2].

There is another deep result which shows that the Maslov class condition in the above theorem is superfluous.

Theorem 3.9 (Abouzaid-Kragh). Let $L$ be a compact exact Lagrangian in $T^{*} M$, then $L$ has vanishing Maslov class. 
This theorem is proved by Abouzaid in an Appendix to Kragh's paper [Kra]. The proof involves ideas from algebraic topology, namely the construction of Viterbo's transfer map on symplectic cohomology as a map of spectra.

Combining the above theorems we obtain the following corollary which will enable our construction of graph selectors in the next section.

Corollary 3.10. Let $L$ be an exact, compact Lagrangian in $T^{*} M$ for $M$ orientable. Then the wrapped Floer cohomology $H W^{*}\left(T_{q}^{*} M, L\right)$ is well defined and

$$
H W^{*}\left(T_{q}^{*} M, L\right) \simeq \mathbb{Z}
$$

Since $H W^{*}\left(T_{q}^{*} M, L\right) \simeq \mathbb{Z}$, we also conclude that $H W_{*}\left(L, T_{q}^{*} M\right) \simeq \mathbb{Z}$.

\section{Graph Selectors}

We will now construct a graph selector for any compact, exact Lagrangian $L$ in $T^{*} M$ when $M$ is orientable. The construction follows the ideas from [Oh1] combined with Corollary 3.10.

4.1. Choice of Hamiltonian $H$ adapted to $L$. Recall the general action integral formula (3.2)

$$
\mathcal{A}_{H}(\gamma)=\int_{[0,1]}\left(\gamma^{*} \theta-H \circ \gamma d t\right)+h_{0}(\gamma(0))-h_{1}(\gamma(1))
$$

associated to the pair

$$
\begin{aligned}
& \left(L_{0}, h_{0}\right)=(L, h) ; \quad L=\iota(N), h=S \circ \iota^{-1} \\
& \left(L_{1}, h_{1}\right)=\left(T_{q}^{*} M, 0\right) .
\end{aligned}
$$

for a fiber $T_{q}^{*} M$ at $q \in M$. Here we note that the constant function $h \equiv 0$ is a primitive of $T_{q}^{*} M$ since $\left.\theta\right|_{T_{q}^{*} M} \equiv 0$.

For the discussion of our main interest in this paper, all the Lagrangian submanifolds $L$ are contained in a compact subset $K$ say, in a disc bundle $D_{R}^{*} M$ for some sufficiently large constant $R>0$. To make our graph selector of $L$ independent of the choice of Hamiltonian $H$ given in $\mathcal{A}_{H}$, we put the following condition on the support of $H$.

Condition 4.1. Let $R>0$ be as above. We assume that

$$
\text { supp } H \subset T^{*} M \backslash D_{R}^{*} M \text {. }
$$

Let $q \in M$ be such that $T_{q}^{*} M$ and $L$ are transversal. By our choice of $H, X_{H}=0$ on $D_{R}^{*} M$ and hence Proposition 3.3 implies that any $x \in \mathcal{X}\left(L, T_{q}^{*} M\right)$ is a constant path associated to an intersection point in $L \cap T_{q}^{*} M$. Therefore we have a one-to-one correspondence

$$
\mathcal{X}\left(L, T_{q}^{*} M\right) \cong T_{q}^{*} M \cap L .
$$

Moreover, since $\theta$ vanishes on $T_{q}^{*} M$, we derive

$$
\mathcal{A}_{H}(x)=h(x)
$$

from (3.2). 
4.2. Structure of the wave front of exact Lagrangians. In this subsection, we give precise description of generic properties of the wave front of exact Lagrangian $(L, h)$ in relation to the singularities of the primitive $h$. We denote the wave front set associated to $(L, h)$ by

$$
W F(L, h)=\left\{(h(x), x) \in \mathbb{R} \times T^{*} M \mid x \in L\right\}
$$

which forms a Legendrian submanifold of the 1-jet bundle $J^{1}(M) \cong \mathbb{R} \times T^{*} M$.

Let $\pi_{L}: L \rightarrow M$ be the restriction to $L$ of the natural projection $\pi: T^{*} M \rightarrow M$. The caustic of $L$ is the set of critical values of $\pi_{L}$, which we denote by $\operatorname{Caus}(L) \subset M$. Let

$$
\mathcal{U}_{L}=M \backslash \operatorname{Caus}(L) \subseteq M .
$$

Note that $\mathcal{U}_{L}$ is the set of $q \in M$ such that $T_{q}^{*} M$ is transversal to $L$.

Next consider a subset of $\mathcal{U}_{L}$ defined as

$$
\mathcal{U}_{L}^{C f}=\left\{q \in \mathcal{U}_{L}|h|_{T_{q}^{*} M \cap L} \text { is injective }\right\}
$$

We remark that although the primitive $h$ is used in their definitions, the sets do not depend on the choice of the primitives (for connected $L$ ). Points in this set are called Cerf-regular, see [Oh5].

The following proposition is an application of Sard's theorem. A similar statement is proved in [Oh5] in a much more complex context, but for convenience of the reader we give a proof here.

Proposition 4.2. Let $L$ be an exact Lagrangian submanifold and $h$ a Liouville primitive. Then $\mathcal{U}_{L}^{C f}$ is an open, dense subset of $M$ of full measure. Furthermore, for any $q \in \mathcal{U}_{L}^{C f}, \pi_{L}^{-1}(q)$ is a finite set $\left\{x_{0}, \ldots, x_{k_{q}}\right\}$ and there exists an open (connected) neighborhood $U_{q}$ of $q$ for which we have the decomposition

$$
\pi_{L}^{-1}\left(U_{q}\right)=\coprod_{i=0}^{k_{q}} V_{x_{i}}
$$

where each $V_{x_{i}}$ is a an open neighborhood of $x_{i}$, and we have

(1) $\left.\pi_{L}\right|_{V_{x_{i}}}: V_{x_{i}} \rightarrow U_{q}$ is a diffeomorphism for each $i=0, \ldots, k_{q}$.

(2) The primitive $h$ restricts to an injective function on each fiber $\pi_{L}^{-1}\left(q^{\prime}\right)$ for all $q^{\prime} \in U_{q}$.

Proof. By definition $\mathcal{U}_{L}$ is the set of regular values of $\pi_{L}$, and hence it is open and has total measure by Sard's theorem. Compactness of $L$ then implies that $\pi_{L}^{-1}(q)$ is a finite set for any $q \in \mathcal{U}_{L}$. Again by definition, $\pi_{L}$ is a local diffeomorphism when restricted to $\mathcal{U}_{L}$, which immediately implies the existence of the neighborhoods $U_{q}$, $V_{x_{0}}, \ldots, V_{x_{k_{q}}}$ and property (i) for any point in $\mathcal{U}_{L}$. Property (ii) follows from the definition of $\mathcal{U}_{L}^{C f}$.

We are left with showing that $\mathcal{U}_{L}^{C f} \subset \mathcal{U}_{L}$ has total measure. For this consider 1 -forms $\varphi_{i}$ on $U_{q}$ for each $i=0, \ldots, k_{q}$, determined by the equations $\left(q^{\prime}, \varphi_{i}\left(q^{\prime}\right)\right)=$ $\left.\pi\right|_{V_{x_{i}}} ^{-1}\left(q^{\prime}\right)$ for $i=0, \ldots, k_{q}$. Denote $x_{i}\left(q^{\prime}\right)=\left(q^{\prime}, \varphi_{i}\left(q^{\prime}\right)\right)$. Then for each pair $0 \leq i<$ $j \leq k_{q}$ define the function $\delta_{i j}: U_{q} \rightarrow \mathbb{R}$ by

$$
\delta_{i j}\left(q^{\prime}\right)=h\left(q^{\prime}, \varphi_{i}\left(q^{\prime}\right)\right)-h\left(q^{\prime}, \varphi_{j}\left(q^{\prime}\right)\right)
$$


and denote its zero set by $\Delta_{i j}=\delta_{i j}^{-1}(0)$. Since $h$ is a Liouville primitive we compute

$$
\begin{aligned}
d_{q^{\prime}}\left(h\left(\cdot, \varphi_{i}(\cdot)\right)\right) & =\left.d h\right|_{x_{i}\left(q^{\prime}\right)} \circ d \pi_{V_{x_{i}\left(q^{\prime}\right)}}^{-1}=\theta_{x_{i}\left(q^{\prime}\right)} \circ d \pi_{V_{x_{i}\left(q^{\prime}\right)}}^{-1} \\
& =p\left(d \pi \circ d \pi_{V_{x_{i}\left(q^{\prime}\right)}}^{-1}\right)=p\left(x_{i}\left(q^{\prime}\right)\right)=\varphi_{i}\left(q^{\prime}\right)
\end{aligned}
$$

for each $i=0, \ldots, k_{q_{0}}$. Therefore for $i \neq j$,

$$
d \delta_{i j}\left(q^{\prime}\right)=\varphi_{i}\left(q^{\prime}\right)-\varphi_{j}\left(q^{\prime}\right) \neq 0
$$

where the last non-vanishing holds by the definition of $\varphi_{i}$ 's. Hence we conclude that the $\Delta_{i j}$ are smooth hyper-surfaces. Then observe

$$
\mathcal{U}_{L}^{C f} \cap U_{q}=U_{q} \backslash \bigcup_{i<j} \Delta_{i j}
$$

hence $\mathcal{U}_{L}^{C f} \cap U_{q}$ is an open set of total measure which implies the desired result.

4.3. Floer-theoretic graph selectors. Consider an exact Lagrangian submanifold $(L, h)$ as before. For each $q \in M$, we define the subset of $\mathbb{R}$,

$$
\operatorname{Spec}(L, h ; q)=\left\{h(x) \in \mathbb{R} \mid x \in L \cap T_{q}^{*} M\right\} .
$$

We call it the spectrum of $(L, h ; q)$. By compactness of $L, \operatorname{Spec}(L, h ; q)$ is a finite set whenever $L$ intersects transversely $T_{q}^{*} M$.

Now we introduce our main

Definition 4.3. For $q \in \mathcal{U}_{L}$ we define the spectral invariant of the pair $T_{q}^{*} M, L$ :

$$
\rho(L, q)=\min \left\{\lambda \in \mathbb{R} \mid\left(j_{\lambda}\right)_{*}: H W_{*}^{\leq \lambda}\left(L, T_{q}^{*} M\right) \rightarrow H W_{*}\left(L, T_{q}^{*} M\right) \text { is surjective }\right\} .
$$

It can be seen, using an argument similar to the one in the construction of (7.3) in Section 7, that these values do not depend on the choice of Hamiltonian $H$ as long as it satisfies the support condition given in Condition 4.1. Since we do not use this fact we will not give a complete proof. Also $\rho(L, q)$ loosely depends on the choice of $h$, not just on $L$. Since the $h-h^{\prime} \equiv$ const for any two generating functions $h, h^{\prime}$ of (connected) $L$, we ignore this dependence.

Remark 4.4. We would like to remark that this definition makes sense for any point $q$ not necessarily in $\mathcal{U}_{L}$, but for these we would have to consider a Hamiltonian that does not satisfy Condition 4.1.

Note that since $H W_{*}\left(L, T_{q}^{*} M\right) \simeq \mathbb{Z}$, the above condition is equivalent to defining $\rho(L, q)$ as

$$
\min _{[\alpha]=\left[\varphi_{*}(1)\right]}\{\nu(\alpha)\}
$$

where $\varphi: \mathbb{Z} \rightarrow C W_{*}\left(L, T_{q}^{*} M\right)$ is any homomorphism that induces an isomorphism on homology. It follows from this interpretation of $\rho(L, q)$ and the support hypothesis (4.1) put on $H$, that the mini-max value $\rho(L, q)$ is realized by the action of some $x_{q} \in L \cap T_{q}^{*} M$, that is, $h\left(x_{q}\right)=\rho(L, q)$. So we conclude

$$
\rho(L, q) \in \operatorname{Spec}(L, h ; q)
$$

for all $q \in \mathcal{U}_{L}$. Therefore $\left(\rho(L, q), x_{q}\right) \in W F(L, h)$ for all $q \in M$. (Such property is called the tightness of the mini-max value, for example see Definition 21.5.2 [Oh6].)

We remark that a priori the definition of $\rho(L, q)$ depends on the family of almost complex structures $\left\{J_{t}\right\}$, used to define the Floer differential. But, by the same argument performed in [Oh1, Lemma 6.3], we can prove the following 
Lemma 4.5. The value $\rho(L, q)$ does not depend on the choice of $\left\{J_{t}\right\}$.

This enables us to define the basic phase function (or Floer theoretic graph selector) $f_{L}: \mathcal{U}_{L} \subseteq M \rightarrow \mathbb{R}$ by $f_{L}(q)=\rho(L, q)$.

The following is the main theorem of this subsection and is the counterpart of Theorem 9.1 in [Oh1]. Note that, in particular, this proves Theorem 1.2 in the Introduction.

Theorem 4.6. Let $L$ be a compact exact Lagrangian and define $f_{L}$ as above. We can extend $f_{L}$ to a Lipschitz function on $M$. If $q \in \mathcal{U}_{L}^{C f}$ then $f_{L}$ is smooth at $q$ and we have

$$
\left(q, d f_{L}(q)\right) \in L \subseteq T^{*} M \text { and } f_{L}(q)=h\left(q, d f_{L}(q)\right) .
$$

Therefore, since $\mathcal{U}_{L}^{C f}$ is an open set of total measure by Proposition 4.2, $f_{L}$ is a graph selector for $L$.

The proof of this theorem will occupy the rest of this subsection. We start with the following proposition whose proof we postpone to Section 7 .

Proposition 4.7. Equip $M$ with a metric and denote by $d$ the associated distance function. Consider $q_{0}, q_{1} \in \mathcal{U}_{L} \subseteq M$ so that $\rho\left(L, q_{0}\right)$ and $\rho\left(L, q_{1}\right)$ is defined. Then

$$
\left|f_{L}\left(q_{0}\right)-f_{L}\left(q_{1}\right)\right| \leq C \cdot d\left(q_{0}, q_{1}\right),
$$

for a constant $C>0$ depending only on $L$ and the Hamiltonian $H$.

We can now extend $f_{L}$ uniquely to a Lipschitz function on $M$ and prove the following

Lemma 4.8. For any $q \in M$, there exists $x_{q} \in L \cap T_{q}^{*} M$ such that $f_{L}(q)=$ $\rho(L, q)=h\left(x_{q}\right)$.

Proof. This is obvious from the construction for points $q \in \mathcal{U}_{L}$. For the other points it follows from an easy continuity argument using denseness of $\mathcal{U}_{L}$ and compactness of $L$. We omit the easy details.

The following stability of tightness of the values $\rho(L, q)$ is a crucial ingredient in the chain level Floer theory entering in the study of spectral invariants. (See [Oh5], especially section 3 thereof, for the illustration of such a usage.)

Proposition 4.9. Consider $q_{0} \in \mathcal{U}_{L}^{C f}$ and take $x_{0} \in L \cap T_{q_{0}}^{*} M$ such that $h\left(x_{0}\right)=$ $f_{L}\left(q_{0}\right)$. Let $V_{x_{0}}$ and $U_{q_{0}}$ be the neighborhoods provided by Proposition 4.2 and for each $q \in U_{q_{0}}$ denote $x_{0}(q):=\left.\pi\right|_{V_{x_{0}}} ^{-1}(q)$. Then we have

$$
f_{L}(q)=h\left(x_{0}(q)\right) \text {. }
$$

Hence, in particular, $f_{L}$ is a smooth function on $U_{q_{0}}$.

Proof. Let $\left\{x_{0}, \ldots, x_{k}\right\}$ be the pre-image of $q_{0}$, consider the data provided by Proposition 4.2 and denote $x_{i}(q)=\left.\pi\right|_{V_{x_{i}}} ^{-1}(q)$, for $i=0, \ldots, k$, so that $x_{i}\left(q_{0}\right)=x_{i}$. We consider the subset

$$
U_{q_{0}}^{=}=\left\{q \in U_{q_{0}} \mid f_{L}(q)=h\left(x_{0}(q)\right)\right\} .
$$

This set is nonempty since $q_{0} \in U_{q_{0}}^{=}$. By continuity of $f$ and $h \circ x_{0}$, it is closed in $U_{q_{0}}$. Now we show that it is also open in $U_{q_{0}}$. Let $q^{\prime} \in U_{q_{0}}^{=}$. By assumption $h$ is injective in $\pi^{-1}\left(q^{\prime}\right)=\left\{x_{0}\left(q^{\prime}\right), \ldots, x_{k}\left(q^{\prime}\right)\right\}$, hence there exists $\epsilon>0$ such that

$$
\left|h\left(x_{i}\left(q^{\prime}\right)\right)-h\left(x_{0}\left(q^{\prime}\right)\right)\right|>\epsilon
$$


for all $i \neq 0$. Since $q^{\prime} \in U_{q_{0}}^{=}$, we have $f_{L}\left(q^{\prime}\right)=h\left(x_{0}\left(q^{\prime}\right)\right)$. Then by continuity of $f_{L}$ and $h\left(x_{i}(-)\right.$ ) (for each $i$ ), we can take a neighborhood $U_{q^{\prime}}^{\prime} \subset U_{q_{0}}$ of $q^{\prime}$, so that $\left|f_{L}(q)-f_{L}\left(q^{\prime}\right)\right|<\epsilon / 2$ for all $q \in U_{q^{\prime}}^{\prime}$ and that $\left|h\left(x_{i}(q)\right)-f_{L}\left(q^{\prime}\right)\right|>\epsilon / 2$ for all $q \in U_{q^{\prime}}^{\prime}$ and $i \neq 0$. By Lemma 4.8, for each $q$ there is some $i$ such that $f_{L}(q)=h\left(x_{i}(q)\right)$. Hence the previous inequalities force $f_{L}(q)=h\left(x_{0}(q)\right)$ for all $q \in U_{q^{\prime}}^{\prime}$ and so $U_{q^{\prime}}^{\prime} \subset U_{q_{0}}^{=}$. This proves that the set $U_{q_{0}}^{=}$is open. Therefore by connectedness of $U_{q_{0}}, U_{q_{0}}^{=}=U_{q_{0}}$, that is, $f_{L}(q)=h\left(x_{0}(q)\right)$ for all $q \in U_{q_{0}}$.

Proof of Theorem 4.6. Proposition 4.7 enables us to extend uniquely $f_{L}$ to a Lipschitz function on $M$. Then Proposition 4.9 implies that $f_{L}$ is a smooth function on $\mathcal{U}_{L}^{C f}$ and that for $q \in \mathcal{U}_{L}^{C f}$ and $x \in L$ such that $f_{L}(q)=h(x)$ we have

$$
\left(q, d f_{L}(q)\right)=x \in L \cap T_{q}^{*} M
$$

To see this, we denote $x=(q, p)$ and compute

$$
d f_{L}(q)=d h \circ d \pi_{V_{x}}^{-1}=\left(\left.\theta\right|_{L}\right)_{x} \circ d \pi_{V_{x}}^{-1}=p\left(d \pi \circ d \pi_{V_{x}}^{-1}\right)=p,
$$

where we have used that $d h=\left.\theta\right|_{L}$ and the definition of $\theta$. This finishes the proof of Theorem 4.6.

Remark 4.10. There might be points $q \in M \backslash \mathcal{U}_{L}^{C f}$ where $f_{L}$ is still differentiable. But for these Equation (4.6) might not hold. However, it follows from Lemma 5.3 that $\left(q, d f_{L}(q)\right)$ lies in the fiberwise convexification of $L$. This fact is the key motivation for the notion of generalized graph selector.

\section{Generalized Graph SeleCtors}

In this subsection we weaken the notion of graph selector, following [BO2], and prove that Lipschitz-exact Lagrangians admit generalized graph selectors.

We start by introducing some notation. For a subset $C \subset T^{*} M$ we denote by $\widehat{C}$ its fiberwise convexification, that is $\widehat{C}_{q}:=\widehat{C} \cap T_{q}^{*} M$ is the convex hull in $T_{q}^{*} M$ of $C \cap T_{q}^{*} M$. Recall that a point in a convex set $C$ is said to be extremal if it is not in the interior of a line segment whose endpoints lie in $C$. Observe that an extremal point in $\widehat{C}$ must lie in $C$.

Definition 5.1. Let $(N, \iota, S)$ be an Lipschitz-exact Lagrangian brane and $L=$ $\iota(N)$. Denote $h=S \circ \iota^{-1}$. A generalized graph selector for $L$ is a Lipschitz function $f: M \rightarrow \mathbb{R}$ satisfying the following conditions,

(a) if $f$ is differentiable at $q$ then $(q, d f(q)) \in \widehat{L}_{q}$;

(b) for each point $q$ where $f$ is differentiable and $d f(q)$ is an extremal point of $\widehat{L}_{q}$ we have $f(q)=h(q, d f(q))$.

Theorem 5.2. Any Lipschitz-exact Lagrangian brane $(N, \iota, S)$, with $N$ compact, $L=\iota(N)$ admits a generalized graph selector.

The proof of this theorem follows closely the proof of Proposition 3 in [BO2] using the graph selector for $L$ constructed in Theorem 4.6. We present the main steps of the proof for the sake of completeness.

The first thing we need is a generalization of a result of non-smooth analysis. 
Lemma 5.3 (Lemma 2 [BO2]). Let $f_{k}: M \rightarrow \mathbb{R}$ be a sequence of equi-Lipschitz functions converging uniformly to a Lipschitz function $f: M \rightarrow \mathbb{R}$. Let $V \subset M$ be a set of total Lebesgue measure such that each one of the $f_{k}$ is differentiable in $V$. We define $\Lambda_{q} \subset T_{q}^{*} M$ to be the set of all limits of subsequences of sequences of the form $\left(q_{k}, d f_{k}\left(q_{k}\right)\right)$ with $\left\{q_{k}\right\} \subset V$ and $q_{k} \rightarrow q$. If $f$ is differentiable at the point $q$ then $(q, d f(q)) \in \widehat{\Lambda}_{q}$.

Proof of Theorem 5.2. Since $(N, \iota, S)$ is an Lipschitz-exact Lagrangian brane we have equi-Lipschitz sequences $S_{k}: N \rightarrow \mathbb{R}$ of smooth functions and $\iota_{k}: N \rightarrow T^{*} M$ of smooth embeddings such that

$$
\iota_{k}^{*} \theta_{k}=d S_{k}, \text { and } S_{k} \rightarrow S, \iota_{k} \rightarrow \iota \text { uniformly. }
$$

As before denote $L_{k}=\iota_{k}(N)$ and consider the Liouville primitive $h_{k}=S_{k} \circ \iota_{k}^{-1}$ : $L_{k} \rightarrow \mathbb{R}$.

Theorem 4.6 gives, for each $\left(N, \iota_{k}, S_{k}\right)$, a graph selector $f_{L_{k}}$ of $L_{k}=\iota_{k}(N)$. Consider $V=\bigcap_{k} \mathcal{U}_{L_{k}}^{C f}$, which is a full measure set where all the $f_{L_{k}}$ are differentiable,

$$
\left(q, d f_{L_{k}}(q)\right) \in L_{k} \text { and } f_{L_{k}}(q)=h_{k}\left(q, d f_{L_{k}}(q)\right),
$$

for all $q \in V$ and all $k$. Since $\left(q, d f_{L_{k}}(q)\right) \in L_{k}$ and $\iota_{k} \rightarrow \iota$ uniformly, there exists a constant $C>0$ independent of $k$ 's but depending only on $L$ such that

$$
\max _{q \in M}\left|d f_{L_{k}}(q)\right| \leq C
$$

and hence $f_{L_{k}}$ are equi-Lipschitz. Then Ascoli-Arzela Theorem implies that the sequence $f_{L_{k}}$ converges uniformly to a Lipschitz limit $f$.

One can now see that $f$ is a generalized graph selector for $(N, \iota, S)$. First we prove that $\Lambda_{q} \subset L_{q}$ which implies $\widehat{\Lambda}_{q} \subset \widehat{L}_{q}$. A point $(q, p)$ in $\Lambda_{q}$ is a limit of a subsequence of the form $\left\{\left(q_{k}, d f_{L_{k}}\left(q_{k}\right)\right)\right\}$ whith $q_{k} \in V, q_{k} \rightarrow q$. By (5.1) we have $\left(q_{k}, d f_{L_{k}}\left(q_{k}\right)\right) \in L_{k}$. Hence there is $x_{k}$ such that $\left(q_{k}, d f_{L_{k}}\left(q_{k}\right)\right)=\iota_{k}\left(x_{k}\right)$ and we can assume $x_{k}$ has a limit $x$. Since $\iota_{k}$ converges uniformly to $\iota$, we have $(q, p)=\lim \iota_{k}\left(x_{k}\right)=\iota(x) \in L_{q}$. Now applying Lemma 5.3 to the sequence $f_{L_{k}}$ we conclude that, if $q$ is a point of differentiability of $f,(q, d f(q))$ lies in $\widehat{\Lambda}_{q}$. Putting these two together shows part (a) of the generalized graph selector statement in its definition.

It remains to see part (b) that $f(q)=h(q, d f(q))$ when $d f(q)$ is extremal in $\widehat{L}_{q}$. Because $\widehat{\Lambda}_{q} \subset \widehat{L}_{q}, d f(q)$ is also extremal in $\widehat{\Lambda}_{q}$ and hence belongs to $\Lambda_{q}$. By the definition of $\Lambda_{q}$ this means there exists a sequence $\left\{q_{k}\right\} \subset V$ such that $\left(q_{k}, d f_{L_{k}}\left(q_{k}\right)\right) \rightarrow(q, d f(q))$. The equality $f(q)=h(q, d f(q))$ now follows from taking the limit $k \rightarrow \infty$ in (5.1).

\section{Applications to Hamiltonian dynamics}

In this section we prove our two applications Theorem 1.3 and Theorem 1.5, following [BO2]. We will concentrate on the differences and when the proofs are straightforward generalizations we omit them.

Let $M$ be a closed orientable $n$-manifold and fix an autonomous $C^{2}$ Tonelli Hamiltonian $H: T^{*} M \rightarrow \mathbb{R}$ (recall this means positive definite Hessian and superlinear in each fiber). As in the introduction let $\mathcal{L}$ be the set of compact Lipschitzexact Lagrangians and given $L=\iota(N) \in \mathcal{L}$ we define the maximal invariant subset 
of $L \cap\{H=a\}$ as

$$
\mathcal{I}_{a}^{*}(L):=\bigcap_{t \in \mathbb{R}} \phi_{H}^{t}(L \cap\{H=a\}) .
$$

We recall the following standard definition from weak KAM Theory.

Definition 6.1. Let $H$ be a Tonelli Hamiltonian on $T^{*} M$. A Lipschitz function $f: M \rightarrow \mathbb{R}$ is called a sub-solution of the (autonomous) Hamilton-Jacobi equation

$$
H(q, d f(q))=a
$$

if it satisfies $H(q, d f(q)) \leq a$ almost everywhere on $M$.

The following regularization result of Bernard, which is proved in this context in Appendix $\mathrm{B}$ of [BO2], plays a significant role in the proof of Theorem 1.3 similarly as in $[\mathrm{BO} 2]$.

Theorem 6.2 (Theorem 3 [BO2]). Given $a \in \mathbb{R}$, if $f$ is a Lipschitz sub-solution of (6.1), then there exists a $C^{1,1}$ sub-solution $\widetilde{f}$ such that $\mathcal{I}_{a}^{*}\left(\Gamma_{f}\right)=\mathcal{I}_{a}^{*}\left(\Gamma_{\tilde{f}}\right)$.

The statement of this theorem is slightly different from that of Theorem 3 [BO2] but is equivalent thereto by the argument given in p.170 [BO2]. Now we have the following

Theorem 6.3. Let $H$ be a Tonelli Hamiltonian and $a \in \mathbb{R}$, then for each $(N, \iota, S) \in$ $\mathcal{L}$ such that $L=\iota(N)$ is contained in the energy sub-level $\{H \leq a\}$, there exists $\Gamma \in \mathcal{G}$ contained in the same energy sub-level $\{H \leq a\}$ and such that

$$
\mathcal{I}_{a}^{*}(\Gamma)=\mathcal{I}_{a}^{*}(L) .
$$

Proof. The proof follows step-by-step the proof of Theorem 1 in [BO2] with the obvious difference that we need to use the new more general version of the generalized graph selector given in Proposition 5.2. Because we start with a compact Lipschitzexact Lagrangian $(N, \iota, S)$ with $L=\iota(N) \subset\{H \leq a\}$, Theorem 5.2 gives a generalized graph selector $f$. Since $H$ is fiberwise convex we have that, whenever defined, $\Gamma_{f} \subset\{H \leq a\}$. Therefore $f$ is a Lipschitz sub-solution of (6.1). Then Theorem 6.2 implies that there exists a $C^{1,1}$ sub-solution $\tilde{f}$ such that $\Gamma_{\tilde{f}} \subset\{H \leq a\}$. One then proves that the graph of $d \tilde{f}$ is what we are looking for, that is $\mathcal{I}_{a}^{*}\left(\Gamma_{\tilde{f}}\right)=\mathcal{I}_{a}^{*}(L)$. The proof of this statement is now the same as the case covered in Section 3 (and page 170) of [BO2]. It uses standard techniques in weak KAM theory so we do not repeat it.

We can now prove Theorem 1.3.

Proof of Theorem 1.3. We first prove that $\alpha_{\mathcal{E}}(H)=\alpha_{\mathcal{G}}(H)$. Recall that

$$
\begin{aligned}
\alpha_{\mathcal{E}}(H) & :=\inf _{L \in \mathcal{E}} \max _{(q, p) \in L} H(q, p), \\
\alpha_{\mathcal{G}}(H) & :=\inf _{L \in \mathcal{G}} \max _{(q, p) \in L} H(q, p) .
\end{aligned}
$$

The inequality $\alpha_{\mathcal{E}}(H) \leq \alpha_{\mathcal{G}}(H)$ is true simply because $\mathcal{G} \subset \mathcal{E}$. Now suppose that $\alpha_{\mathcal{E}}(H)<\alpha_{\mathcal{G}}(H)$. Then there is a $\left.c \in\right] \alpha_{\mathcal{E}}(H), \alpha_{\mathcal{G}}(H)[$ such that the set $\{H \leq c\}$ contains an element $L \in \mathcal{E}$. Then by Theorem 6.3 there exists a $\Gamma \in \mathcal{G}, \Gamma \subset\{H \leq c\}$ which contradicts the definition of $\alpha_{\mathcal{G}}(H)$. Combining the two we have proved $\alpha_{\mathcal{E}}(H)=\alpha_{\mathcal{G}}(H)$. 
The coincidence of the definitions for the Aubry and Mañé sets is now a direct consequence of the definitions themselves, the coincidence of the critical values and Theorem 6.3.

The remaining equalities, for when $L \in \mathcal{L}$, are proven in the exact same way since Theorem 6.3 applies to these Lagrangians.

Before we prove the second application we need

Lemma 6.4. Consider $(N, \iota, S) \in \mathcal{L}$ such that $L=\iota(N)$ is invariant under the flow of a Tonelli Hamiltonian $H$. Then there exists e such that $L \subset\{H=e\}$.

Proof. Since $\iota$ is Lipschitz and $H$ is $C^{2}, H \circ \iota$ is Lipschitz and hence differentiable almost everywhere. Proposition 2.5 implies there is a set $Z$ of total measure where $\iota^{*} \omega=0$ and both $\iota$ and $S$ are differentiable. Let $x \in Z$, then

$$
d(H \circ \iota)(x)=d H(\iota(x)) d \iota(x)=\omega\left(X_{H}(\iota(x)), d \iota(x)\right) .
$$

Since $\phi_{H}^{t}(L) \subset L$ we know $X_{H}(\iota(x)) \subset T_{\iota(x)} L$. Therefore, since $\iota^{*} \omega=0$ in $Z$, we have

$$
\omega\left(X_{H}(\iota(x)), d \iota(x)\right)=0 .
$$

This implies that $H \circ \iota$ is a constant function, which means that there exists $e$ such that $L \subset\{H=e\}$.

Proof of Theorem 1.5. Since $L=\iota(N)$ is invariant under the flow of $H$, Lemma 6.4 gives $e$ such that $L \subset\{H=e\}$. Therefore, since $\mathcal{I}_{e}^{*}(L)$ is the maximal invariant subset of $L \cap\{H=e\}$ and $L$ is invariant we have

$$
\mathcal{I}_{e}^{*}(L)=L .
$$

Now by Theorem 6.3 there exists a $\Gamma \in \mathcal{G}$ such that

$$
\mathcal{I}_{e}^{*}(L)=\mathcal{I}_{e}^{*}(\Gamma) \subset \Gamma .
$$

Hence $L \subset \Gamma$ and since $M$ is connected we conclude $L=\Gamma$.

\section{LIPSCHITZ CONTINUITY OF THE GRAPH SELECTOR}

In this section, we give the proof of Proposition 4.7. The proof follows the ideas of Section 12.5 [Oh6] restricted to the case of submanifold $S=\{q\}$, but now in the wrapped context. The main step is to construct a chain map $\Phi: C W_{*}\left(L, T_{q_{0}}^{*} M\right) \rightarrow$ $C W_{*}\left(L, T_{q_{1}}^{*} M\right)$ which induces an isomorphism in homology.

Remark 7.1. Here we provide full details of this proof because Section 12.5 [Oh6] (and [Oh1]) is written in the unwrapped setting and because the construction of the homotopy map used in Section 12.5 [Oh6] (which follows Nadler's argument $[\mathrm{N}])$ is different from that of the original construction of [Oh1]). Also some of the details of this proof are not given in [Oh6].

We start by taking a minimizing geodesic $c:[0,1] \rightarrow M$ from $q_{0}$ to $q_{1}$ with length $\ell=d\left(q_{0}, q_{1}\right)$. We then consider a small generic perturbation of the path, again denoted by $c$, and extend the derivative $c^{\prime}$ into an ambient vector field $X$ supported in a small neighborhood $V$ of the image of $c$. We may choose $X$ so that

$$
\max _{q \in M}|X(q)| \leq \ell+\epsilon
$$


where $\epsilon>0$ can be chosen arbitrarily small. Consider the autonomous Hamiltonian $G^{0}=\langle p, X(q)\rangle$ and denote by $\phi_{G^{0}}^{s}$ the associated flow. Observe that this defines a Hamiltonian isotopy from $T_{q_{0}}^{*} M$ to $T_{q_{1}}^{*} M$, in fact $\phi_{G^{0}}^{s}\left(T_{q_{0}}^{*} M\right)=T_{c(s)}^{*} M$.

We will deform this isotopy to another isotopy from $T_{q_{0}}^{*} M$ to $T_{q_{1}}^{*} M$ by expressing it as the composition of two isotopies in the following way. We choose $R_{1}>R+1$ (as defined in (4.1)) such that $H$ is quadratic outside $D_{R_{1}}^{*} M$. Let $\tilde{\chi}: \mathbb{R} \rightarrow[0,1]$ be a cut-off function satisfying

$$
\widetilde{\chi}(t)= \begin{cases}0, & t \leq R \\ 1, & t \geq R_{1}\end{cases}
$$

and set $\chi=1-\tilde{\chi}$. Let $\phi_{\tilde{G}}^{s}$ be the Hamiltonian flow associated to $\tilde{G}(q, p)=$ $\tilde{\chi}(|p|)\langle p, X(q)\rangle$ and denote $\tilde{N}_{s}=\phi_{\tilde{G}}^{s}\left(T_{q_{0}}^{*} M\right)$. From the construction it follows that $\widetilde{N}_{s}$ is constant on $D_{R}^{*} M$. Similarly we define the Hamiltonian

$$
G=\chi(|y|)\langle p, X(q)\rangle
$$

and denote by $\phi_{G}^{s}$ the associated Hamiltonian flow. We define $N_{s}=\phi_{G^{1}}^{s}\left(\widetilde{N}_{1}\right)$ and note that $N_{s}$ is constant on $T^{*} M \backslash D_{R_{1}}^{*} M$ and coincides with $T_{c(s)}^{*} M$ on $D_{R}^{*} M$, moreover $N_{1}=T_{q_{1}}^{*} M$ and $N_{0}=\widetilde{N}_{1}$.

Note that, since $L \subset D_{R}^{*} M$, the intersection points $L \cap \tilde{N}_{s}$ are independent of $s \in[0,1]$. Therefore there is an obvious isomorphism of vector spaces

$$
\Phi_{0}: C W_{*}\left(L, T_{q_{0}}^{*} M\right) \rightarrow C W_{*}\left(L, \tilde{N}_{1}\right)
$$

Furthermore $\Phi_{0}$ is a map of chain complexes. To see this we consider the following parametrized moduli spaces

$$
\mathcal{M}^{\text {par }}\left(x_{0}, x_{1}\right):=\bigcup_{s \in[0,1]}\{s\} \times \mathcal{M}^{s}\left(x_{0}, x_{1}\right),
$$

where $\mathcal{M}^{s}\left(x_{0}, x_{1}\right)$ is the moduli space defined in (3.5) but with boundary conditions in $L$ and $\tilde{N}_{s}$. For a generic choice of the almost complex structure $\mathcal{M}^{\text {par }}\left(x_{0}, x_{1}\right)$ becomes a compact smooth one-dimensional cobordism between $\mathcal{M}^{0}\left(x_{0}, x_{1}\right)$ and $\mathcal{M}^{1}\left(x_{0}, x_{1}\right)$. Thus we conclude $\# \mathcal{M}^{0}\left(x_{0}, x_{1}\right)=\# \mathcal{M}^{1}\left(x_{0}, x_{1}\right)$ and therefore $\Phi_{0}$ is a map of chain complexes leaving the filtration unchanged.

Now we will define a chain map $\Phi_{1}: C W_{*}\left(L, N_{0}\right) \rightarrow C W_{*}\left(L, T_{q_{1}}^{*} M\right)$ by considering the Cauchy-Riemann equation with moving boundary conditions.

We fix a smooth elongation function $\rho: \mathbb{R} \rightarrow[0,1]$ such that

$$
\rho(\tau)= \begin{cases}0 & \tau \leq 0, \\ 1 & \tau \geq 1,\end{cases}
$$

and $\rho^{\prime}(\tau)>0$ on $(0,1)$. In particular, we have $\operatorname{supp} \rho^{\prime} \subset[0,1]$.

Now we consider

$$
\left\{\begin{array}{l}
\frac{\partial u}{\partial \tau}+J_{t}\left(\frac{\partial u}{\partial t}-X_{H}(u)\right)=0 \\
u(\tau, 0) \in L, u(\tau, 1) \in N_{\rho(\tau)}
\end{array}\right.
$$

Given $x_{0} \in \mathcal{X}\left(L, N_{0}\right)$ and $x_{1} \in \mathcal{X}\left(L, T_{q_{1}}^{*} M\right)$ we denote by $\mathcal{N}\left(x_{0}, x_{1}\right)$ the set of maps $u$ satisfying the above equation and converging (exponentially) to $x_{0}$ at $-\infty$ and to $x_{1}$ at $+\infty$. (Here there is no $\mathbb{R}$-action and so we do not quotient out the moduli space). It again follows from the (strong) maximum principle proved in Section 
7.3 [AS] (see alternatively [Ab3, Lemma 3.3]) that solutions of (7.4) satisfy a $C^{0}$ bound. More precisely we have the following lemma.

Lemma 7.2. If $u \in \mathcal{N}\left(x_{0}, x_{1}\right)$ then $u(\tau, t) \in D_{R_{1}}^{*} M$ for any $(\tau, t)$.

This is stated in [AS] just for the Cauchy-Riemann equations with fixed boundary conditions, but because our boundary conditions are fixed outside $D_{R_{1}}^{*} M$ the same proof applies.

For generic $\left\{J_{t}\right\}$, when $\left|x_{0}\right|=\left|x_{1}\right|$, the moduli space $\mathcal{N}\left(x_{0}, x_{1}\right)$ is a smooth oriented manifold of dimension 0 . Gromov compactness together with the above $C^{0}$ bound then implies that $\mathcal{N}\left(x_{0}, x_{1}\right)$ is compact and therefore we can define the signed count $\# \mathcal{N}\left(x_{0}, x_{1}\right)$. We then define the map

$$
\Phi_{1}\left(x_{0}\right)=\sum_{x_{1} \in \mathcal{X}\left(L, T_{q_{1}}^{*} M\right),} \# \mathcal{| x _ { 0 } | = | x _ { 1 } |} \# \mathcal{N}\left(x_{0}, x_{1}\right) \cdot x_{1} .
$$

The proof that this is a chain map which induces an isomorphism in homology is a standard argument in Floer theory that applies to both the wrapped or unwrapped context. We refer the reader to Section 12.5 [Oh6] for complete details. Putting these maps together we obtain a chain map $\Phi=\Phi_{1} \circ \Phi_{0}: C W_{*}\left(L, T_{q_{0}}^{*} M\right) \rightarrow$ $C W_{*}\left(L, T_{q_{1}}^{*} M\right)$, which induces an isomorphism $\Phi_{*}$ in homology.

Now we study the action of $\Phi$ on the filtration $\nu$.

Lemma 7.3. Given $x_{0} \in \mathcal{X}\left(L, N_{0}\right), x_{1} \in \mathcal{X}\left(L, T_{q_{1}}^{*} M\right)$ and $u \in \mathcal{N}\left(x_{0}, x_{1}\right)$ we have

$$
\mathcal{A}_{H}\left(x_{1}\right)-\mathcal{A}_{H}\left(x_{0}\right)=-\int_{\mathbb{R}} \int_{[0,1]}\left|\frac{\partial u}{\partial t}-X_{H}(u)\right|_{J_{t}}^{2} d t d \tau+\int_{\mathbb{R}} \rho^{\prime}(\tau) G(u(\tau, 1)) d \tau,
$$

where $|-|_{J_{t}}$ is the induced metric $\omega\left(-, J_{t}-\right)$.

Assuming this lemma for the moment, we complete the proof of Proposition 4.7. Lemma 7.3 immediately implies

$$
\mathcal{A}_{H}\left(x_{1}\right)-\mathcal{A}_{H}\left(x_{0}\right) \leq \int_{-\infty}^{\infty} \rho^{\prime}(\tau) G(u(\tau, 1)) d \tau \leq \max _{\tau \in \mathbb{R}} G(u(\tau, 1)) .
$$

It follows from the definition $G(q, p)=\chi(|p|)\langle p, X(q)\rangle$ and (7.1) that

$$
\max _{\tau \in \mathbb{R}} G(u(\tau, 1)) \leq R_{1}\left(d\left(q_{0}, q_{1}\right)+\epsilon\right) .
$$

This gives the following key inequality

$$
\mathcal{A}_{H}\left(x_{1}\right)-\mathcal{A}_{H}\left(x_{0}\right) \leq R_{1}\left(d\left(q_{0}, q_{1}\right)+\epsilon\right) .
$$

The rest of the argument is standard in the study of any type of spectral invariant. The chain map $\Phi_{0}$ preserves the filtration and therefore we only need to study what happens for $\Phi_{1}$. The inequality (7.5) implies that if $\nu\left(x_{0}\right) \leq \lambda$ then $\nu\left(\Phi_{1}\left(x_{0}\right)\right) \leq \lambda+\delta$, where $\delta=R_{1}\left(d\left(q_{0}, q_{1}\right)+\epsilon\right)$. Therefore the map $\Phi_{1}$ induces the following commutative diagram

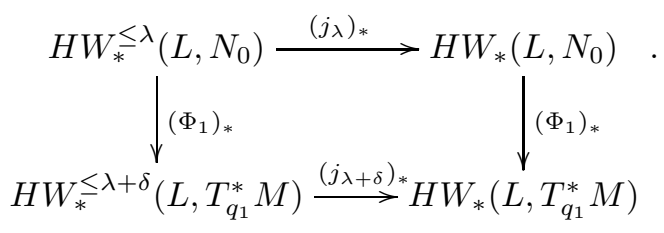


Since $\left(\Phi_{1}\right)_{*}$ on the right hand side is an isomorphism, we conclude

$$
\rho\left(L, q_{1}\right) \leq \rho\left(L, q_{0}\right)+R_{1}\left(d\left(q_{0}, q_{1}\right)+\epsilon\right) .
$$

Since $\epsilon$ is arbitrary we conclude $\rho\left(L, q_{1}\right) \leq \rho\left(L, q_{0}\right)+R_{1} d\left(q_{0}, q_{1}\right)$. By changing the role of $q_{0}$ and $q_{1}$, we prove the other side of the inequality, which leads to

$$
\left|f_{L}\left(q_{1}\right)-f_{L}\left(q_{0}\right)\right| \leq R_{1} d\left(q_{0}, q_{1}\right),
$$

since by definition $f_{L}(q)=\rho(L, q)$. This completes the proof of the proposition modulo the proof of Lemma 7.3.

Proof of Lemma 7.3. We define the following parametrized version of the action functional (defined in (3.2)):

$$
\mathcal{A}_{H, s}(\gamma)=\int_{[0,1]}\left(\gamma^{*} \theta-H \circ \gamma d t\right)+h(\gamma(0))-h_{s}(\gamma(1))
$$

for $\gamma \in \mathcal{P}\left(L, N_{s}\right)$. Here $h_{s}: N_{s} \rightarrow \mathbb{R}$ is the Liouville primitive of $N_{s}$. Recall that $N_{s}=\phi_{G}^{s}\left(\tilde{N}_{1}\right)$ and denote $S_{s}=h_{s} \circ \phi_{G}^{s}: \tilde{N}_{1} \rightarrow \mathbb{R}$. An elementary computation (see for example [Oh6, Proposition 3.4.8]) gives the following formula:

$$
S_{s}=\tilde{h}+\int_{0}^{s}\left(\left\langle\theta, X_{G}\right\rangle-G\right) \circ \phi_{G}^{t} d t
$$

where $\tilde{h}$ is the Liouville primitive for $\tilde{N}_{1}$. Note that, by construction both $\tilde{N}_{s}$ and $N_{s}$ agree with cotangent fibers inside $D_{R}^{*} M$. Hence this formula shows that $\tilde{h}$ and $h_{s}$ are always zero inside $D_{R}^{*} M$. Therefore, since $N_{1}=T_{q_{1}}^{*} M, h_{1}$ must be zero by uniqueness up to constant of the Liouville primitive. In this way, for $x_{1} \in \mathcal{X}\left(L, T_{q_{1}}^{*} M\right)$ we have $\mathcal{A}_{H, 1}\left(x_{1}\right)=\mathcal{A}_{H}\left(x_{1}\right)$ and for $x_{0} \in \mathcal{X}\left(L, N_{0}\right)$ we have $\mathcal{A}_{H, 0}\left(x_{0}\right)=\mathcal{A}_{H}\left(x_{0}\right)$. Therefore for $u \in \mathcal{N}\left(x_{0}, x_{1}\right)$ we have

$$
\mathcal{A}_{H}\left(x_{1}\right)-\mathcal{A}_{H}\left(x_{0}\right)=\int_{-\infty}^{\infty} \frac{d}{d \tau}\left(\mathcal{A}_{H ; \rho(\tau)}(u(\tau))\right) d \tau .
$$

We compute

$$
\begin{aligned}
\frac{d}{d \tau}\left(\mathcal{A}_{H ; \rho(\tau)}(u(\tau))\right)= & \frac{d}{d \tau}\left(\int_{[0,1]}(u(\tau))^{*} \theta-H(u(\tau, t)) d t+h(u(\tau, 0))\right) \\
& -\frac{d}{d \tau}\left(h_{\rho(\tau)}(u(\tau, 1))\right) .
\end{aligned}
$$

For the first summand, we use the first variation with free boundary condition (see Equation (2.17) [Oh1] for example) together with the fact that $u$ is a solution of (7.4) and get

$$
-\int_{[0,1]}\left|\frac{\partial u}{\partial t}-X_{H}(u)\right|_{J_{t}}^{2} d t+\left\langle\theta, \frac{\partial u}{\partial \tau}(\tau, 1)\right\rangle .
$$

For the second, we start by noticing that the boundary condition $u(\tau, 1) \in N_{\rho(\tau)}$ implies that $u(\tau, 1)=\phi_{G}^{\rho(\tau)}(v(\tau))$ for some curve $v(\tau) \in \tilde{N}_{1}$. Hence $h_{\rho(\tau)}(u(\tau, 1))=$ $S_{\rho(\tau)}(v(\tau))$ and using 7.8 we compute

$$
\begin{array}{r}
\frac{d}{d \tau}\left(h_{\rho(\tau)}(u(\tau, 1))\right)=d S_{\rho(\tau)}\left(\frac{d v}{d \tau}\right)+\left.\rho^{\prime}(\tau)\left(\frac{d S_{s}}{d s}\right)\right|_{s=\rho(\tau)}(v(\tau)) \\
=d h_{\rho(\tau)} \circ d \phi_{G}^{\rho(\tau)}\left(\frac{d v}{d \tau}\right)+\rho^{\prime}(\tau)\left(\left\langle\theta, X_{G}\right\rangle\left(\phi_{G}^{\rho(\tau)}(v(\tau))\right)-G\left(\phi_{G}^{\rho(\tau)}(v(\tau))\right)\right) .
\end{array}
$$


It follows from the definitions that

$$
\frac{\partial u}{\partial \tau}(\tau, 1)=d \phi_{G}^{\rho(\tau)}\left(\frac{d v}{d \tau}\right)+\rho^{\prime}(\tau) X_{G}(u(\tau, 1)) .
$$

Plugging this in the previous equation and using the definition of Liouville primitive we obtain

$$
\begin{aligned}
\frac{d}{d \tau}\left(h_{\rho(\tau)}(u(\tau, 1))\right)= & d h_{\rho(\tau)}\left(\frac{\partial u}{\partial \tau}(\tau, 1)\right)-\rho^{\prime}(\tau) d h_{\rho(\tau)}\left(X_{G}(u(\tau, 1))\right) \\
& +\rho^{\prime}(\tau)\left\langle\theta, X_{G}\right\rangle(u(\tau, 1))-\rho^{\prime}(\tau) G(u(\tau, 1)) \\
= & \left\langle\theta, \frac{\partial u}{\partial \tau}(\tau, 1)\right\rangle-\rho^{\prime}(\tau) G(u(\tau, 1)) .
\end{aligned}
$$

Combining the above calculations we conclude

$$
\frac{d}{d \tau}\left(\mathcal{A}_{H ; \rho(\tau)}(u(\tau))\right)=-\int_{[0,1]}\left|\frac{\partial u}{\partial t}-X_{H}(u)\right|_{J_{t}}^{2} d t+\rho^{\prime}(\tau) G(u(\tau, 1)),
$$

which immediately proves the lemma.

We have now finished the proof of Proposition 4.7.

\section{REFERENCES}

[Ab1] M. Abouzaid. A cotangent fibre generates the Fukaya category. Adv. Math. 228, no. 2 (2011) $894-939$.

[Ab2] M. Abouzaid. Nearby Lagrangians with vanishing Maslov class are homotopy equivalent. Invent. Math. 189, no. 2 (2012) 251 - 313.

[Ab3] M. Abouzaid. On the Wrapped Fukaya category and based loops. J. Symplectic Geom. 10, no. 1 (2012) 27-79.

[AS] M. Abouzaid and P. Seidel. An open string analogue of Viterbo functoriality. Geometry E Topology. 14 (2010) $627-718$.

[Arn] M.-C. Arnaud. On a theorem due to Birkhoff. Geom. Funct. Anal. 120 (2010) 1307 1316.

[Be] P. Bernard. Symplectic Aspects of Mather theory. Duke Math. J. 136, no. 3 (2007) 401 $-420$.

[Be1] P. Bernard. Existence of $C^{1,1}$ critical sub-solutions of the Hamilton-Jacobi equations on compact manifolds. Ann. Sci. l'Ecole Norm. Sup. 40, no. 3 (2007) $445-452$.

[BO1] P. Bernard and J. Oliveira dos Santos. A geometric definition of the Aubry-Mather set. J. Top. Anal. 2, no. 3 (2010) $385-393$.

[BO2] P. Bernard and J. Oliveira dos Santos. A geometric definition of the Mañé-Mather set and a Theorem of Marie-Claude Arnaud. Math. Proc. Camb. Phil. Soc. 152 (2012) 167 -178 .

[BS] L. Buhovsky and S. Seyfaddini. Uniqueness of generating Hamiltonians for continuous Hamiltonian flows. J. Symp. Geom. 11, no. 1 (2013) $37-52$.

[C] M. Chaperon. Lois de conservation et géométrie symplectique. Comptes rendus de l'Académie des sciences. Série 1, Mathématique 312, no. 4 (1991) 345 - 348.

[dR] G. de Rham. Differentiable Manifolds, A Series of Comp. Studies in Math. 266, Springer Verlag, Berlin-Heidelberg-New York, 1984.

[El] Y. Eliashberg. A theorem on the structure of wave fronts and its application in symplectic topology (in Russian). Funkstsional. Anal. i Prilozhen. 21, no. 3 (1987) 65 - 72.

[EG] L. Evans and R. Gariepy. Measure Theory and Fine Properties of Functions. (Studies in Advanced Math., CRC Press, New York, 1992).

[Fe] H. Federer. Geometric Measure Theory, Classics in Math. Springer-Verlag, BerlinHeidelberg-New York, 1969.

[FOOO] K. Fukaya, Y.-G. Oh, H. Ohta and K. Ono. Lagrangian intersection Floer theory-anomaly and obstruction I - II. (AMS/IP Studies in Advanced Mathematics, vol 46, Amer. Math. Soc./International Press, 2009). 
[FSS] K. Fukaya, P. Seidel and I. Smith. Exact Lagrangian submanifolds in simply-connected cotangent bundles. Invent. Math. 172 (2008) $1-27$.

[Hor] L. Hörmander. Fourier integral operators I. Acta Math. 127 (1971) 79 - 183.

[HLS] V. Humiliére, R. Leclerq and S. Seyfaddini. Coisotropic rigidity and $C^{0}$-symplectic geometry. Duke Math. J. 164, no. 4 (2015) $767-799$.

[KO] R. Kasturirangan and Y.-G. Oh. Floer homology of open subsets and a relative version of Arnold's conjecture. Math. Z. 236, no. 1 (2001) $151-189$.

[Kra] T. Kragh. Parametrized ring-spectra and the nearby Lagrangian conjecture. Geometry E Topology 17, no. 2 (2013) $639-731$.

[LauS] F. Laudenbach and J.-C. Sikorav. Persistence of intersection with the zero section during a Hamiltonian isotopy into a cotangent bundle. Invent. Math 82, no. 2 (1985) 349 - 357.

[Mu S. Müller. The group of Hamiltonian homeomorphisms in the $L^{\infty}$-norm. J. Korean Math. Soc. 45, no. 6 (2008) $1769-1784$.

[N] D. Nadler. Microlocal branes are constructible sheaves. Selecta Math. 15, no. 4 (2009) $563-619$.

[Oh1] Y.-G. Oh. Symplectic topology as the geometry of action functional, I. J. Differ. Geom. 46 (1997) $499-577$.

[Oh2] Y.-G. Oh. Symplectic topology as the geometry of action functional, II. Commun. Anal. Geom. 7 (1999) $1-55$.

[Oh3] Y.-G. Oh. Construction of spectral invariants of Hamiltonian paths on closed symplectic manifolds in "The Breadth of Symplectic and Poisson Geometry". Prog. Math. 232 (Birkhäuser, Boston, 2005) 525 - 570 .

[Oh4] Y.-G. Oh. Locality of continuous Hamiltonian flows and Lagrangian intersection with the conormal of open subsets. J. Gökova Geom. Top. 1 (2007) 1 - 32.

[Oh5] Y.-G. Oh. Floer mini-max theory, the Cerf diagram, and the spectral invariants. $J$. Korean Mah. Soc. 46 (2009) $363-447$.

[Oh6] Y.-G. Oh. Symplectic Topology and Floer Homology I 83 II. (New Mathematical Monographs No. 28 \& 29, Cambridge University Press, Cambridge, 2015).

[OM] Y.-G. Oh and S. Müller. The group of Hamiltonian homeomorphisms and $C^{0}$ symplectic topology. J. Symp. Geom. 5 (2007) $167-219$.

[PPS] BibliographyG. Paternain, L. Polterovich and K. Siburg. Boundary rigidity for Lagrangian submanifolds, non-removable intersections, and Aubry-Mather theory. Mosc. Math. J. 3, no. 2 (2003) $593-619$.

[Se] P. Seidel. Fukaya Categories and Picard-Lefschetz Theory. (Zürich Lec. Advanced Math., European Math. Soc., Zürich, 2008).

[Sik] J. C. Sikorav. Problémes d'intersections et de points fixes en géométrie hamiltonienne. Comment. Math. Helv. 62 (1987) $62-73$.

[V1] C. Viterbo. Symplectic topology as the geometry of generating functions. Math. Ann. 292 (1992) $685-710$.

[V2] C. Viterbo. On the uniqueness of generating Hamiltonian for continuous limits of Hamiltonian flows. Internat. Math. Res. Notices, (2006) Article ID 34028. Erratum, ibid, (2006), Article ID 38784.

Department of Mathematics and Statistics, Boston University, USA

E-mail address: lamorim@bu.edu

Center for Geometry and Physics, Institute for Basic Sciences (IBS), Pohang 37673 , Korea \& Department of Mathematics, POStech, Pohang 37673, KOREA

E-mail address: yongoh1@postech.ac.kr

Department of Mathematics and Statistics, Boston University, USA

E-mail address: jamorim@bu.edu 